\title{
Pareto Optimal Design of the Tuned Mass Damper
}

\author{
Ahmed Abed*, Om El Khaiat Moustachi \\ Mohammadia School of Engineering, University Mohammed V in Rabat, Morocco \\ *Corresponding Author: ahmed_abed.93@outlook.com
}

\begin{abstract}
Received September 28, 2021; Revised November 24, 2021; Accepted December 13, 2021
Cite This Paper in the following Citation Styles

(a): [1] Ahmed Abed, Om El Khaiat Moustachi, "Pareto Optimal Design of the Tuned Mass Damper," Civil Engineering and Architecture, Vol.10, No.1, pp. 236-245, 2022. DOI: 10.13189/cea.2022.100120

(b): Ahmed Abed, Om El Khaiat Moustachi, (2022). Pareto Optimal Design of the Tuned Mass Damper. Civil Engineering and Architecture, 10(1), $236-245$. DOI: $10.13189 /$ cea.2022.100120
\end{abstract}

Copyright $@ 2022$ by authors, all rights reserved. Authors agree that this article remains permanently open access under the terms of the Creative Commons Attribution License 4.0 International License

\begin{abstract}
Strong vibrations due to wind induced loads and earthquakes have undesirable effects on tall buildings and long-span bridges, and can cause discomfort, dizziness and anxiety for their occupants and users. The Single Tuned Mass Damper (STMD) is vibration control device used to mitigate strong motion. This study investigated the STMD from the perspective of multi-objective optimization. The main goal was to identify and characterize the Pareto set of optimal STMD parameters in terms of damping and stiffness. The optimization was performed using a multi-objective optimization algorithm based on gradient descent and successive bisecting of the search domain. $H_{\infty}$ and $H_{2}$ norms of the transfer matrices for structural displacement and acceleration were considered as design objectives. We found that for peak response reduction, irrespective of the excitation type, structural damping and STMD mass ratio, the Pareto set was linear and extended mainly in the direction of frequency ratio, while the damping ratio varied marginally within the set. For RMS displacement and peak acceleration minimization, the Pareto set was composed of two segments. Additionally, in all instances, the Pareto set was found to be bounded by the single-objective optimal STMDs for the considered design objectives. The implications of the findings were discussed and approximations of the Pareto optimal STMD were suggested.
\end{abstract}

Keywords Pareto Optimal, Tuned Mass Damper, Multiobjective, Vibration, Control, Optimization

\section{Introduction}

Vibration control has become a requirement for the design of secure and reliable tall buildings and long-span bridges. In fact, with the advancement of construction materials and meth- ods, the stiffness of tall and long structures has declined. As a result, structures became more prone to dynamic effects caused by earthquakes and wind induced loads. Moreover, strong vibrations not only threatened the structural integrity of buildings and bridges, but also the comfort of users and occupants [1]. In this regard, vibration control is considered one of the costeffective solutions to mitigate such problems. Subsequently, a wide range of control strategies and devices were researched and developed during the last century [2], and the Single Tuned Mass Damper (STMD) is one of the most well known and mature [3].

The optimization of the STMD has considerable impact on the effectiveness of the control system. For a given STMD mass, optimal damping and stiffness improve the capacity of the STMD in mitigating structural vibration. As such, multiple researchers investigated the optimal STMD and its behavior. First, Den Hartog [4] formulated the fixed-point theory to derive expressions for optimal STMD parameters. Later, various closed form expressions of optimal STMD parameters for Single Degree Of Freedom (SDOF) undamped structures under different load types and for various optimized parameters were proposed by Warburton [5]. Researchers also studied the STMD in the case of damped structures [6-8]. Recently, evolutionary algorithms and machine learning were successfully employed for the optimization of the STMD for multiple degrees of freedom structures [9-13]. While optimal STMD is effective, Xu and Igusa [14] revealed that it's sensitive to changes in frequency ratio, which may decrease the control system performance. They also proposed the multiple tuned mass dampers as an alternative and robust control system. The mentioned studies investigated various aspects of the STMD, nevertheless, they only considered the design and study of this control device from the perspective of single objective optimization.

The optimization of the STMD is a Multi-objective Optimization Problem (MOP). The objectives of vibration control are ensuring the structural integrity of structures, the security 
of their various services and the comfort of the users and occupants. To satisfy these different requirements, designers are often tasked with optimizing the STMD for multiple design objectives at the same time. Such objectives may include, peak displacement, peak acceleration and inter-story drift. For example, to ensure the comfort of occupants and users, excessive motion must be mitigated, and peak acceleration values, that induce inertial forces on the human body, should be reduced [1]. To evaluate the STMD performance in mitigating structural vibration and measure structural response, especially displacement and acceleration, the norms $H_{\infty}$ and $H_{2}$ are generally used. $H_{\infty}$ represents the peak value of the dynamic amplification factor of the considered response component. $\mathrm{H}_{2}$, on the other hand, can be interpreted as the average system gain taken over all frequencies, and is also the Root-Mean-Square (RMS) of the impulse response of the system.

While it's possible to convert an MOP into a single-objective optimization problem, for example by considering a linear combination of normalized displacement and acceleration as a design objective, it is not guaranteed that the obtained design is Pareto optimal. In this sense, multi-objective optimization is advantageous as it can generate a set of optimal designs, from which designers can choose an adequate design based on some constraints. While, recent studies have examined the STMD design as an MOP under multiple conditions $[15,16]$, to the best knowledge of the authors, no study has explored and characterized the set of Pareto optimal solutions for the design of the STMD as an MOP.

The main goal of this study was to investigate the behavior of the Pareto optimal STMD, and characterize the Pareto set of optimal designs for structural vibration mitigation. The controlled structure was modeled as a Single Degree Of Freedom (SDOF) system. $\mathrm{H}_{\infty}$ and $\mathrm{H}_{2}$ norms of displacement and acceleration were considered as design objectives. The multiobjective optimization was performed using the algorithm proposed by Dellnitz, Schütze and Hestermeyer [17]. An efficient implementation of the algorithm was coded in Julia. Finally, approximations of the Pareto sets were proposed.

\section{Problem formulation}

A Single Degree Of Freedom (SDOF) structure with mass $m_{s}$, damping $c_{s}$, stiffness $k_{s}$, damping ratio $\xi_{s}$ and natural frequency $\omega_{s}$ is equipped with an STMD with mass $m_{d}$, damping $c_{d}$, stiffness $k_{d}$ and natural frequency $\omega_{d}$. The vibration control system is characterized by its mass ratio $\mu_{d}$, damping ratio $\xi_{d}$ and frequency ratio $\gamma_{d}$.

$$
\mu_{d}=\frac{m_{d}}{m_{s}}, \quad \xi_{d}=\frac{c_{d}}{2 m_{d} \omega_{d}}, \quad \gamma_{d}=\frac{\omega_{d}}{\omega_{s}}
$$

\subsection{Transfer functions}

The governing system of equations of the motion of the structure-STMD system (Figure 1) is written in matrix form as

$$
\mathbf{M} \ddot{\mathbf{X}}+\mathbf{C} \dot{\mathbf{X}}+\mathbf{K X}=\mathbf{U}
$$

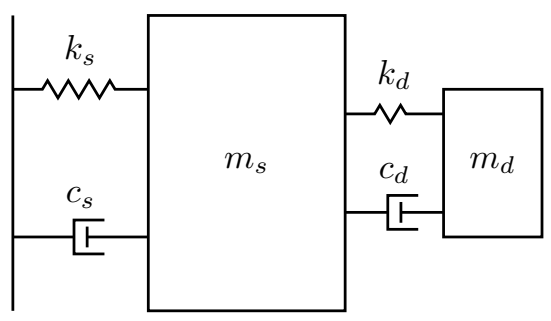

Figure 1. Structure-STMD combined system.

where $\mathbf{X}=\left[\begin{array}{ll}x_{s} & x_{d}\end{array}\right]^{T}$ is the displacement vector. $x_{s}$ and $x_{d}$ are the primary structure and damper displacements relative to ground. $\ddot{\mathbf{X}}$ and $\dot{\mathbf{X}}$ are the acceleration and velocity vectors. $\mathbf{M}$, $\mathbf{C}$, and $\mathbf{K}$ are the mass, damping and stiffness matrices of the combined system.

$$
\begin{aligned}
& \mathbf{M}=\left[\begin{array}{cc}
m_{s} & 0 \\
0 & m_{d}
\end{array}\right] \\
& \mathbf{C}=\left[\begin{array}{cc}
c_{s}+c_{d} & -c_{d} \\
-c_{d} & c_{d}
\end{array}\right] \\
& \mathbf{K}=\left[\begin{array}{cc}
k_{s}+k_{d} & -k_{d} \\
-k_{d} & k_{d}
\end{array}\right]
\end{aligned}
$$

where $m_{s}, c_{s}$ and $k_{s}$ are the mass, damping and stiffness of the primary structure.

$\mathbf{U}$ is the external loading vector written as

$$
\mathbf{U}=u\left[\begin{array}{ll}
m_{s} & \Theta m_{d}
\end{array}\right]^{T}
$$

where $u$ is the external force acting on the structure, and $\Theta$ specifies the excitation type: $\Theta=0$ for wind induced load, and $\Theta=1$ for seismic excitation.

Under harmonic excitation, the vector transfer function of the combined system has for expression

$$
\begin{aligned}
\mathbf{G}(\omega) & =\left[\begin{array}{c}
G_{d}(\omega) \\
G_{s t m d}(\omega)
\end{array}\right] \\
& =\left(-\omega^{2} \mathbf{M}+i \omega \mathbf{C}+K\right)^{-1}\left[\begin{array}{c}
m_{s} \\
\Theta m_{d}
\end{array}\right]
\end{aligned}
$$

where $G_{d}$ and $G_{s t m d}$ are the transfer functions for the main structure and STMD displacements, respectively. $\omega$ is the excitation frequency, and $i$ is the imaginary number.

Considering the relationship between displacement and acceleration in frequency domain $\ddot{x}=-\omega^{2} x$, the acceleration transfer function is defined.

$$
G_{a}(\omega)=\omega^{2} G_{d}(\omega)
$$

We also define the transfer functions for the structure without control as $G_{d}^{w / o}$ for displacement and $G_{a}^{w / o}$ for acceleration.

$$
\begin{aligned}
G_{d}^{w / o} & =\frac{1}{-\omega^{2} m_{s}+i \omega c_{s}+k_{s}} \\
G_{a}^{w / o} & =\frac{\omega^{2}}{-\omega^{2} m_{s}+i \omega c_{s}+k_{s}}
\end{aligned}
$$




\subsection{Design Objectives}

The $H_{\infty}$ and $H_{2}$ norms of a transfer function $G$ are defined as

$$
\begin{aligned}
H_{\infty}(G) & =\sup _{\omega \geq 0}|G(\omega)| \\
H_{2}(G) & =\left(\frac{1}{2 \pi} \int_{-\infty}^{\infty}|G(\omega)|^{2} \mathrm{~d} \omega\right)^{1 / 2}
\end{aligned}
$$

The $H_{\infty}$ and $H_{2}$ of the transfer functions $G_{d}, G_{a}, G_{d}^{w / o}$ and $G_{a}^{w / o}$ can be defined in the same manner. It should be noted that for the acceleration response, only the $H_{\infty}$ norm was considered.

For the controlled structure

$$
\begin{aligned}
H_{\infty}\left(G_{d}\right) & =H_{\infty}^{d} \\
H_{2}\left(G_{d}\right) & =H_{2}^{d} \\
H_{\infty}\left(G_{a}\right) & =H_{\infty}^{a}
\end{aligned}
$$

For the uncontrolled structure

$$
\begin{aligned}
H_{\infty}\left(G_{d}^{w / o}\right) & =H_{\infty}^{d w / o} \\
H_{2}\left(G_{d}^{w / o}\right) & =H_{2}^{d w / o} \\
H_{\infty}\left(G_{a}^{w / o}\right) & =H_{\infty}^{a w / o}
\end{aligned}
$$

The primary objective of vibration control is limiting structural response, and more specifically, the displacement and acceleration components. The goal is to guarantee that support elements, like columns and beams, can withstand the external load. Additionally, it seeks to prevent the failure of the different building services, such as plumbing and ventilation. A successful controller design must also ensure the occupants and users comfort, and avert feelings of stress and panic that are usually induced by excessive motion.

The performance of the control system is defined by its ability to reduce structural response. In this paper, we considered the $H_{\infty}$ and $H_{2}$ norms of displacement and the $H_{\infty}$ of acceleration of the primary structure as potential design objectives.

The current MOP can be stated as follows

$$
\begin{array}{ll}
\text { given } & \xi_{s}, \Theta, \mu_{d} \\
\text { find } & x=\left(\xi_{d}, \gamma_{d}\right) \in \mathbb{R}^{2} \\
\text { minimize } & F(x)
\end{array}
$$

where $F$ is 2 dimensional vector-valued function. $F$ represents the multi-objective for the current optimization problem of the STMD. In this paper we considered the two cases of $F=\left(f_{\infty}^{d}, f_{\infty}^{a}\right)$ and $F=\left(f_{2}^{d}, f_{\infty}^{a}\right)$ such that

$$
\begin{aligned}
f_{\infty}^{d} & =\frac{H_{\infty}^{d}}{H_{\infty}^{d w / o}} \\
f_{2}^{d} & =\frac{H_{2}^{d}}{H_{2}^{d w / o}} \\
f_{\infty}^{a} & =\frac{H_{\infty}^{a}}{H_{\infty}^{a w / o}}
\end{aligned}
$$

\section{Optimization procedure}

The main objective of the optimization procedure of the MOP is to identify the Pareto set. In other words, the domain of STMD parameters that generates the Pareto front for the considered design objectives. For any point in this domain, any change in one of the parameters causes an increase in at least one of the design objectives.

The proposed algorithm is based on the paper written by Dellnitz, Schütze and Hestermeyer [17]. Given a starting search domain, the goal of the algorithm is to identify the Pareto set contained in that domain for the considered design objectives. The procedure produces a tight covering of the Pareto set represented by a collection of boxes containing the points of the Pareto set.

\subsection{Pareto optimality}

In the case of multi-objective optimization, the objective function is not a scalar but a vector, it is therefore necessary to properly define the notion of minimum for a vector. In accordance with the definition of Pareto efficiency, the following definition was adopted

$$
\forall a, b \in \mathbb{R}^{2} \quad a \leq b \Longleftrightarrow a_{i} \leq b_{i} \text { for } i=1,2
$$

In this sense, a point $\bar{x} \in \mathbb{R}^{2}$ is called globally Pareto optimal, or non-dominated, if there is no point $y \in \mathbb{R}^{2}$ such that $F(y) \leq F(\bar{x})$ and $F(y) \neq F(\bar{x})$.

\subsection{Search direction}

The proposed optimization scheme relies on gradient descent to minimize the objective function. The search direction for every optimization objective was taken as the gradient of the considered objective function with respect to STMD parameters $\xi_{d}$ and $\gamma_{d}$.

To determine the search direction that minimizes all objectives, the set of optimization objectives $F$ is associated with the following Quadratic Optimization Problem (QOP)

$$
\begin{array}{ll}
\text { given } & F=\left(f_{1}, f_{2}\right), x \\
\text { find } & \alpha=\left(\alpha_{1}, \alpha_{2}\right) \in \mathbb{R}^{+2} \text { such that } \sum_{i=1}^{2} \alpha_{i}=1 \\
\text { minimize } & \left\|\sum_{i=1}^{2} \alpha_{i} \nabla f_{i}(x)\right\|^{2}
\end{array}
$$

where $F$ is the multi-objective, $f_{1}$ and $f_{2}$ are the design objectives described in 2.2, $x$ is the point at which we want to find the search direction, $\nabla f_{i}(x)$ is the search direction for the i-th optimization objective calculated at $x$.

If $\bar{\alpha}=\left(\overline{\alpha_{1}}, \overline{\alpha_{2}}\right)$ denotes the solution of the QOP, then, the global search direction $q(x)$ at $x \in \mathbb{R}^{2}$ is found by

$$
q(x)=-\sum_{i=1}^{2} \bar{\alpha}_{i} \nabla f_{i}(x)
$$

Given a starting point $x_{i}$ in the search domain, the next point $x_{i+1}$ which minimizes all optimization objectives is calculated 
by the iteration

$$
x_{i+1}=x_{i}+h q\left(x_{i}\right)
$$

where $h$ is the step size.

After a sufficient number of iterations, the described scheme converges to a sub-stationary point $\bar{x}$ for which $q(\bar{x}) \approx 0$. This means the goal of the optimization procedure is to identify the set of sub-stationary points.

\subsection{Step size}

While the iteration scheme may converge to a sub-stationary point, its performance depends on the step size $h$. On the one hand, a large step size decreases convergence time, however, it may cause overshooting. On the other hand, a small step size reduces the risk of overshooting but increases convergence time. Therefore, a variable step size which takes into account the shape of the objective functions was adopted.

At every step of the iteration scheme, the step size was computed with line search [20]. First, for every optimization objective $f_{i}$, we defined the following minimization objective

$$
\phi\left(s_{i}\right)=f_{i}\left(x+s_{i} q(x)\right)
$$

where $s_{i}$ is the step size, $x$ is the point at which we found the search direction and $q(x)$ is the search direction found by solving the QOP.

To calculate a step size that guarantees the minimization of the objective $f_{i}$, we proceeded as follows

1. Set the initial step size $s_{i}=1.0$, and other parameters $c_{1}=10^{-4}, c_{2}=0.1$ and $\tau=0.9$.

2. Terminate if $s_{i}$ satisfies the strong Wolfe conditions

$$
\begin{aligned}
& \phi\left(s_{i}\right) \leq \phi(0)+c_{1} s_{i} \phi^{\prime}(0) \\
& \left|\phi^{\prime}\left(s_{i}\right)\right| \leq c_{2}\left|\phi^{\prime}(0)\right|
\end{aligned}
$$

3. Else, reduce the step size

$$
s_{i} \leftarrow \tau s_{i}
$$

Repeat from 2

The step sizes $s_{1}$ and $s_{2}$ minimize the two optimization objectives $f_{1}$ and $f_{2}$, respetively. To ensure that all objectives are minimized, the step size $h$ for the MOP was taken as the smallest of the computed step sizes.

$$
h=\min \left(s_{1}, s_{2}\right)
$$

\subsection{Bisecting}

The initial search domain, denoted by $B_{0}$, is considered to be a box defined by its center and radiuses, one for each of the dimensions. Subsets of $B_{0}$ are considered to be a collection of boxes and are obtained through bisecting $B_{0}$ along one of the dimensions. For example, $B_{1}$ refers to the subsets of $B_{0}$ that are obtained by bisecting along the first dimension. Subsets of $B_{1}$ are obtained in the same manner by bisecting along the next dimension. In general, $B_{k}$ refers to the subsets of $B_{0}$ that

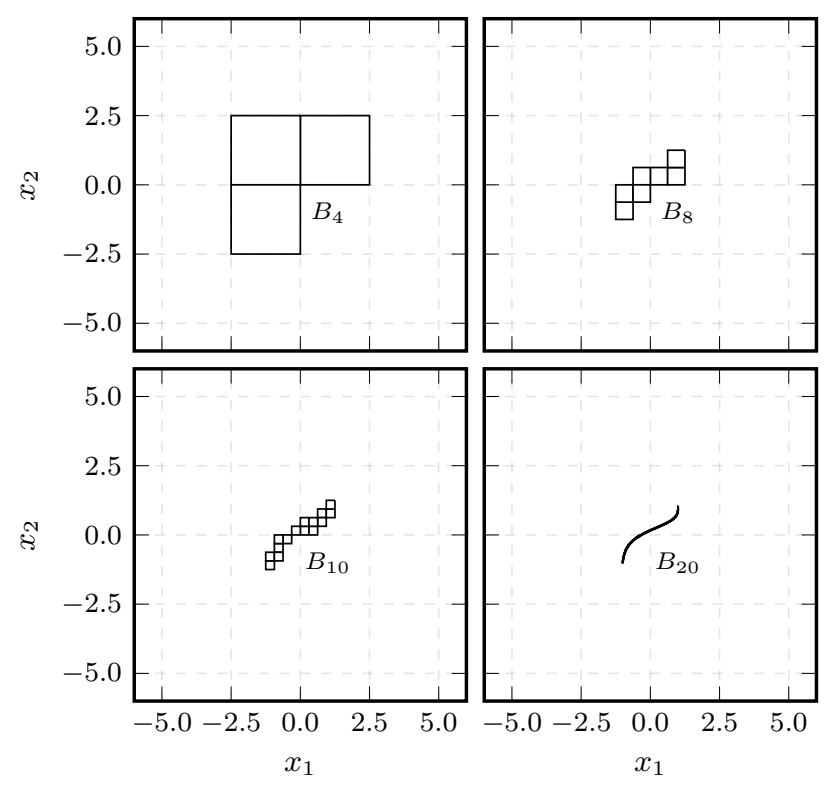

Figure 2. Coverings of the Pareto set for the multi-objective $F(x)=$ $\left(\left(x_{1}-1\right)^{2}+\left(x_{2}-1\right)^{4},\left(x_{1}+1\right)^{2}+\left(x_{2}+1\right)^{2}\right)$, produced by the implemented algorithm. $B_{k}$ is the collection of boxes that covers the Pareto set at bisecting depth $k$. The initial search domain is $B_{0}=[-5,5] \times[-5,5]$ as given in [17].

are obtained after $k$ operations of bisecting. $k$ is called the bisecting depth. It should be noted that after bisecting along the last dimension, the next bisecting direction is the first dimension. Figure 2 shows how the algorithm iteratively converges to cover the Pareto set for one of the optimization problems described in [17].

\subsection{Implementation}

Let $B_{0}$ be the initial box which covers the Pareto optimal set. The subsequent collections of boxes are constructed in the following manner

1. Through bisecting of $B_{i-1}$, a new collection $\hat{B}_{i}$ of boxes is created such that

$$
\bigcup_{B \in B_{i-1}} B=\bigcup_{B \in \hat{B}_{i}} B
$$

2. The new collections of boxes $B_{i}$ is defined as

$$
B_{i}=\left\{B \in \hat{B}_{i} / B \cap g\left(B_{i-1}\right) \neq \emptyset\right\}
$$

where $g(x)$ is the image of the point $x$ after applying the iteration scheme a sufficient number of times starting from $x$.

The algorithm was coded with the Julia programming language. The following Julia scientific packages were used:

- Optim.j1 [18] for single valued function minimization;

- FiniteDifferences.jl for numerical evaluation of the gradient; 
- NLopt.jl [19] for quadratic optimization;

- LineSearches.jl for step size calculation using line search;

- ControlSystems.jl for computing $H_{\infty}$ and $H_{2}$ norms;

- LsqFit.jl for line fitting in the least squares sense.

\subsection{Samples}

In the second step, the described algorithm relies on evaluating the image of a set. In practice, this is achieved by computing the images of an adequate number of randomly selected sample points. At the start of the optimization process, the boxes are large, therefore, a considerable number of points, more than 64 for each box, must be used to accurately evaluate their images. However, near the end of the process, the size of the boxes becomes so small that only one point per box is needed.

\subsection{Initial search domain}

The described algorithm is only able to cover the Pareto set within the given initial search domain. If the initial search domain doesn't cover the entirety of the Pareto set, then the results of the algorithm won't be complete. For the studied problem, which is the optimization of the STMD, the values of the optimized parameters have known bounds. As such, we safely assumed that the damping ratio $\xi_{d}$ is contained in the range $[0.0,1.0]$, and the frequency ratio $\gamma_{d}$ is in the range $[0.0,2.0]$. By starting from $B_{0}=[0.0,1.0] \times[0.0,2.0]$ and running the algorithm for a few iterations, we had an approximate location of the Pareto set and adjusted the initial search domain accordingly.

\section{Results}

The implemented algorithm was employed to optimize damping and frequency ratios of the STMD for peak displacement and acceleration minimization. Additionally, the impact of STMD mass ratio, structural damping and excitation type on the Pareto set was also investigated.

\subsection{Peak displacement and acceleration}

In this section, we examined and characterized the Pareto set for peak displacement and acceleration reduction under seismic excitation. The following values of structural damping and the mass ratio of the STMD were selected: $\xi_{s}=0.03$ and $\mu_{d}=0.02$. The bisecting depth was set to 16 , and the initial search domain to $B_{0}=[0.0,0.2] \times[0.9,1.0]$.

The Pareto set and Pareto front were both linear (Figure 3). The Pareto set was found to be extended in the direction of frequency ratio.

The single-objective optimization of the STMD showed that the designs that minimize either $f_{\infty}^{d}$ or $f_{\infty}^{a}$ were located at the extremities of the Pareto set. They differed only in frequency ratio, and had almost equal damping ratios. The design from the Pareto set with minimal frequency ratio $\left(\xi_{d}=\right.$
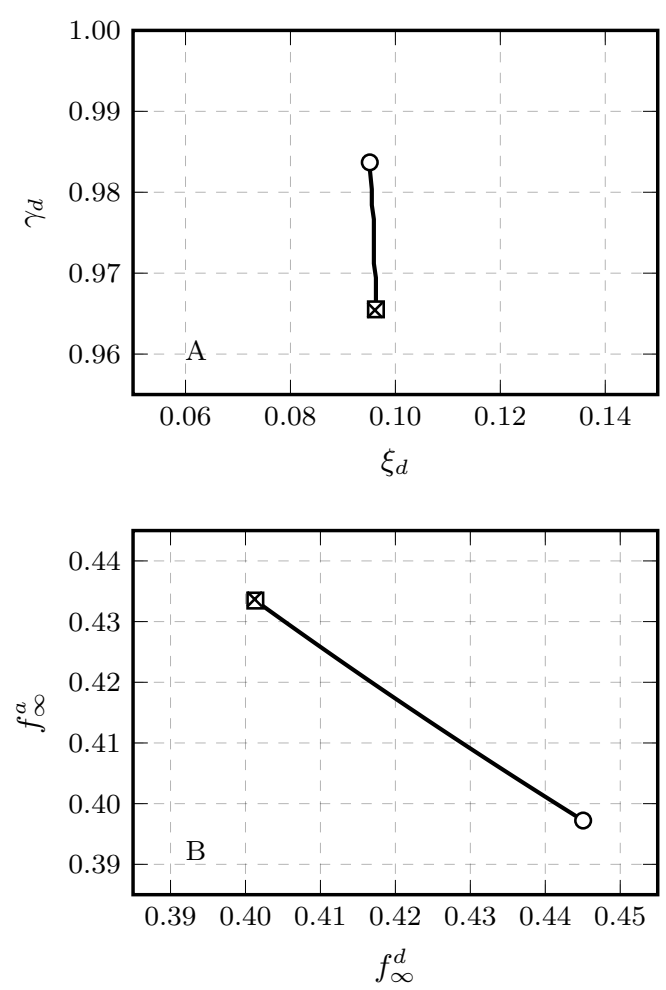

Figure 3. (A) Pareto set and (B) Pareto front for normalized peak displacement and acceleration reduction for $\xi_{s}=0.03, \mu_{d}=0.02$ under seismic excitation. $\square$ The optimal design for $f_{\infty}^{d}$. O The optimal design for $f_{\infty}^{a}$. $\times$ The optimal design for $f_{\infty}^{d}+f_{\infty}^{a}$.

$0.096, \gamma_{d}=0.9657$ ) was optimal for the first design objective $f_{\infty}^{d}$, while the STMD design with maximal frequency ratio $\left(\xi_{d}=0.095, \gamma_{d}=0.9839\right)$ was optimal for the second design objective $f_{\infty}^{a}$.

The slope of the Pareto front was -0.827 , which indicated that the considered design objectives were competing objectives, and the reduction in peak displacement was higher than the reduction of peak acceleration across the Pareto set (Fig. 3B).

The single-objective optimization of the combined objective $f_{\infty}^{d}+f_{\infty}^{a}$ was not successful in finding the Pareto optimal solution of the MOP. In fact, it produced a design that was optimal only for peak displacement reduction.

\section{Effect of $\mu_{d}, \xi_{s}$ and $\Theta$ on the Pareto set}

In this section, we investigated the effect of the different parameters that influence STMD design on the Pareto set, mainly mass ratio, structural damping ratio and external load (Figure 4). The case of $\xi_{s}=0.03$ and $\mu_{d}=0.02$ under seismic excitation (Fig. 4A) was taken as reference.

Mass ratio: The reduction of mass ratio caused the Pareto set to move moderately leftwards and upwards (Fig. 4B). In other words, the optimal damping ratio decreased and optimal frequency ratio increased. Additionally, the range of optimal frequency ratio decreased, and the Pareto set shrunk.

Structural damping: With the increase of structural damping ratio, the Pareto set shifted slightly rightwards (Fig. 4C). 

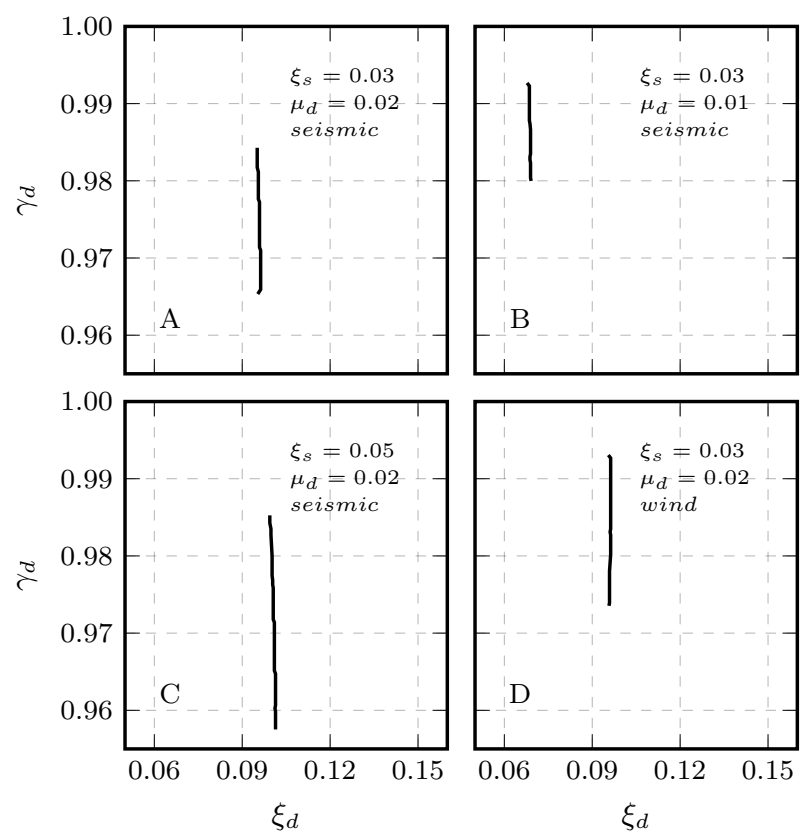

Figure 4. Pareto sets for normalized peak displacement and acceleration reduction for different parameters combinations.

The optimal damping ratio rose by a small amount. In terms of optimal frequency ratio, the maximal value of this parameter remained almost the same, while the minimal value decreased. As a result, the range of optimal frequency ratio grew, and the Pareto set expanded.

External excitation: Under wind induced load, the Pareto set moved upwards compared to the case under seismic excitation, and rotated slightly clockwise (Fig. 4D). The values of optimal damping ratio remained almost the same. The optimal frequency ratio increased, however, the length of the range of this parameter didn't change.

\section{Parametric study}

Regardless of structural damping ratio, STMD mass ratio and excitation type, the Pareto set for the minimization of peak displacement and acceleration was almost linear (Figure 5). Moreover, it extended in the direction of frequency ratio. Put differently, the value of Pareto optimal damping ratio of the STMD remained almost constant inside the Pareto set.

In all instances, the Pareto set for $F=\left(f_{\infty}^{d}, f_{\infty}^{a}\right)$ was located between the single-objective optimal designs for $f_{\infty}^{d}$ and $f_{\infty}^{a}$. It was bounded from the bottom by the single-objective optimal design for peak displacement reduction, and from the top by the one for peak acceleration reduction.

\subsection{RMS displacement and peak acceleration}

In this case, we examined and characterized the Pareto set for peak and RMS displacement reduction under seismic excitation. We considered the same previous design problem: $\xi_{s}=0.03$ and $\mu_{d}=0.02$. The bisecting depth was set to 16 , and the initial search domain to $B_{0}=[0.0,0.2] \times[0.9,1.0]$.
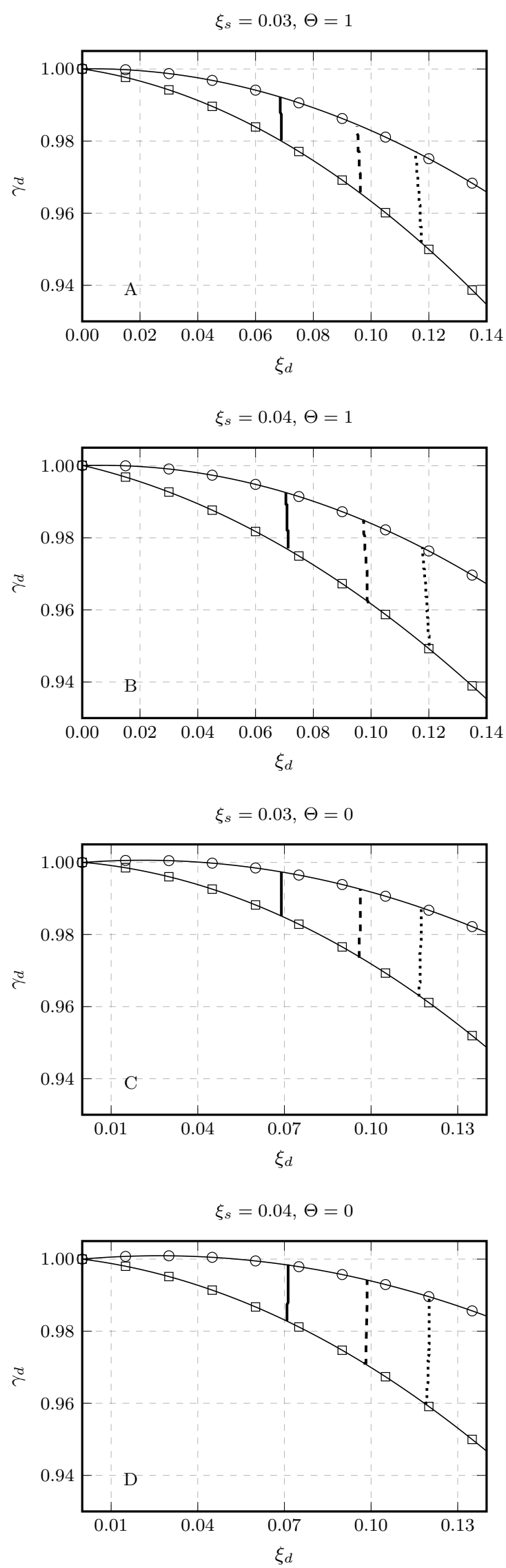

Figure 5. Pareto sets for peak displacement and acceleration reduction for $\xi_{s}=0.03$ and $\xi_{s}=0.04$ under seismic $(\Theta=1)$ and wind induced $(\Theta=0)$ loads. $-\mu_{d}=0.01,--\mu_{d}=0.02, \cdots \mu_{d}=0.03$. $\square$ Optimal design for $f_{\infty}^{d}$. $\bigcirc$ Optimal design for $f_{\infty}^{a}$. 

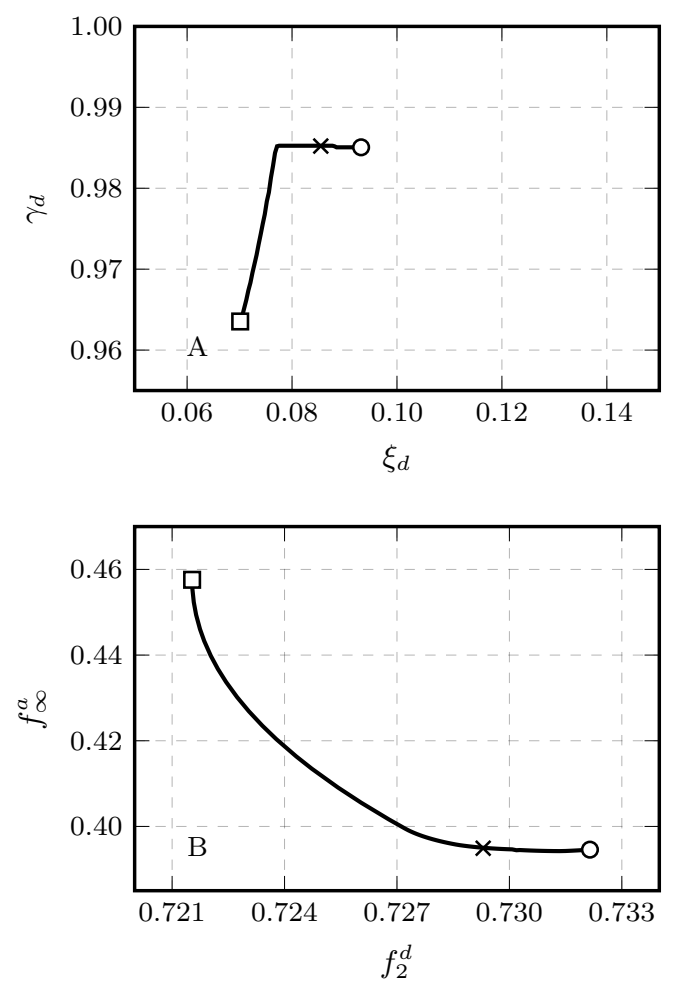

Figure 6. (A) Pareto set and (B) Pareto front for normalized RMS displacement and peak acceleration reduction for $\xi_{s}=0.03, \mu_{d}=0.02$ under seismic excitation. $\square$ The optimal design for $f_{2}^{d}$. ○ The optimal design for $f_{\infty}^{a}$. $\times$ The optimal design for $f_{2}^{d}+f_{\infty}^{a}$.

Contrary the previous study, the Pareto set and Pareto front were not linear. The Pareto set for the minimization of RMS displacement and peak acceleration was composed of two segments (Fig. 6A). The Pareto front had the same shape as a reciprocal function (Fig. 7B).

Similar to the results of 4.1, the Pareto set joined the singleobjective optimal STMD designs for $f_{2}^{d}$ and $f_{\infty}^{a}$. The design with minimal damping and frequency ratios $\left(\xi_{d}=0.070, \gamma_{d}=\right.$ $0.9635)$ was optimal for RMS displacement, and the one with maximal damping and frequency ratios $\left(\xi_{d}=0.093, \gamma_{d}=\right.$ 0.9851) minimized peak acceleration. Starting from the optimal design for $f_{2}^{d}$ and traveling along the Pareto set, the frequency ratio increased considerably in the beginning, while damping ratio increased moderately. Afterwards, the frequency ratio remained constant as the damping ratio increased until it reached the optimal design for $f_{\infty}^{a}$.

The shape of the Pareto front (Fig. 6B) shows that the considered design objectives were competing objectives. Moreover, the reduction in peak acceleration was much higher than that of RMS displacement across the Pareto set.

The single-objective optimization of the combined objective $f_{2}^{d}+f_{\infty}^{a}$ was not successful in finding a true Pareto optimal design, but instead, it minimized only peak acceleration.

\section{Effect of $\mu_{d}, \xi_{s}$ and $\Theta$ on the Pareto set}

In this section, we investigated the effect of mass ratio, structural damping ratio and external load on the Pareto set (Fig-
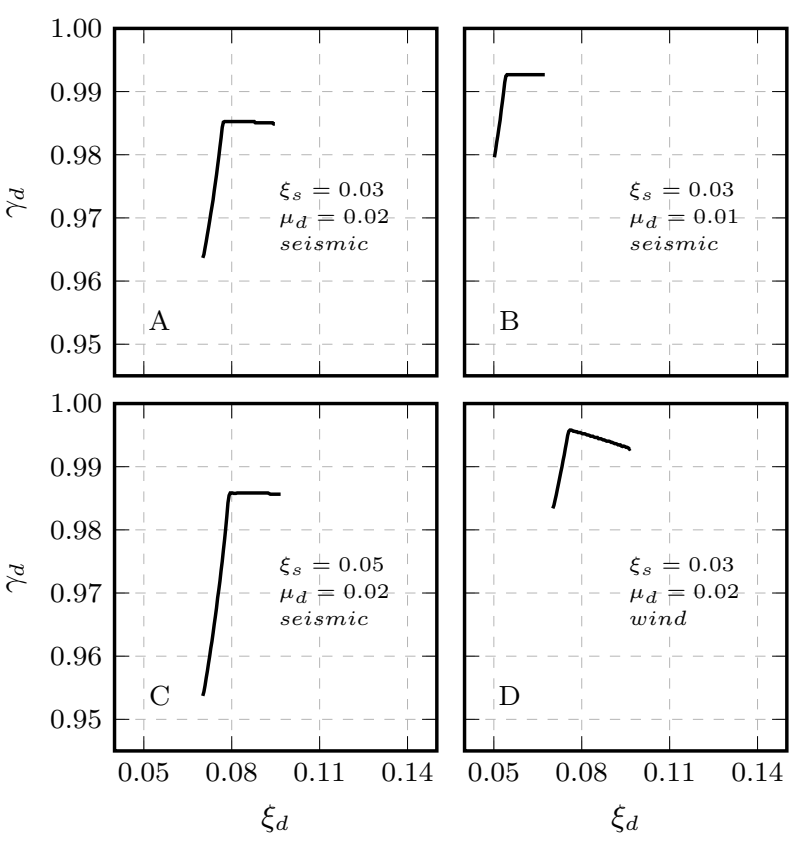

Figure 7. Pareto sets for normalized RMS displacement and peak acceleration reduction for different parameters combinations.

ure 7). The case of $\xi_{s}=0.03$ and $\mu_{d}=0.02$ under seismic excitation (Fig. 7A) was taken as reference.

Mass ratio: As the mass ratio decreased, the Pareto set moved upwards and leftwards (Fig. 7B). The optimal damping ratio declined and optimal frequency ratio increased. In addition, the range of Pareto optimal frequency ratio shrunk.

Structural damping: With the increase of damping ratio, the Pareto set shifted marginally upwards and rightwards (Fig. 7C). It also extended significantly downwards, in other words, the minimal value of Pareto optimal frequency ratio decreased, and the range of this parameter expanded.

External excitation: Under wind induced load, the Pareto set moved upwards (Fig. 7D). The extremities of the Pareto set had the same value of damping ratio as before, however, the values of frequency ratio rose, but not by the same amount. As a result, the Pareto set shrunk, and its shape changed slightly.

\section{Parametric study}

Regardless of the value of structural damping ratio and mass ratio, the Pareto set, for RMS displacement and peak acceleration reduction, maintained its shape under either external excitation (Figure 8).

In all cases, the Pareto set for $F=\left(f_{2}^{d}, f_{\infty}^{a}\right)$, was located between the single-objective optimal designs for $f_{2}^{d}$ and $f_{\infty}^{a}$. It was bounded from the bottom by the single-objective optimal design for RMS displacement reduction, and from the top by the one for peak acceleration reduction.

\section{Design formulas}

While the optimization for $F=\left(f_{\infty}^{d}, f_{\infty}^{a}\right)$ produced a linear Pareto set which can be easily approximated as it was bounded 

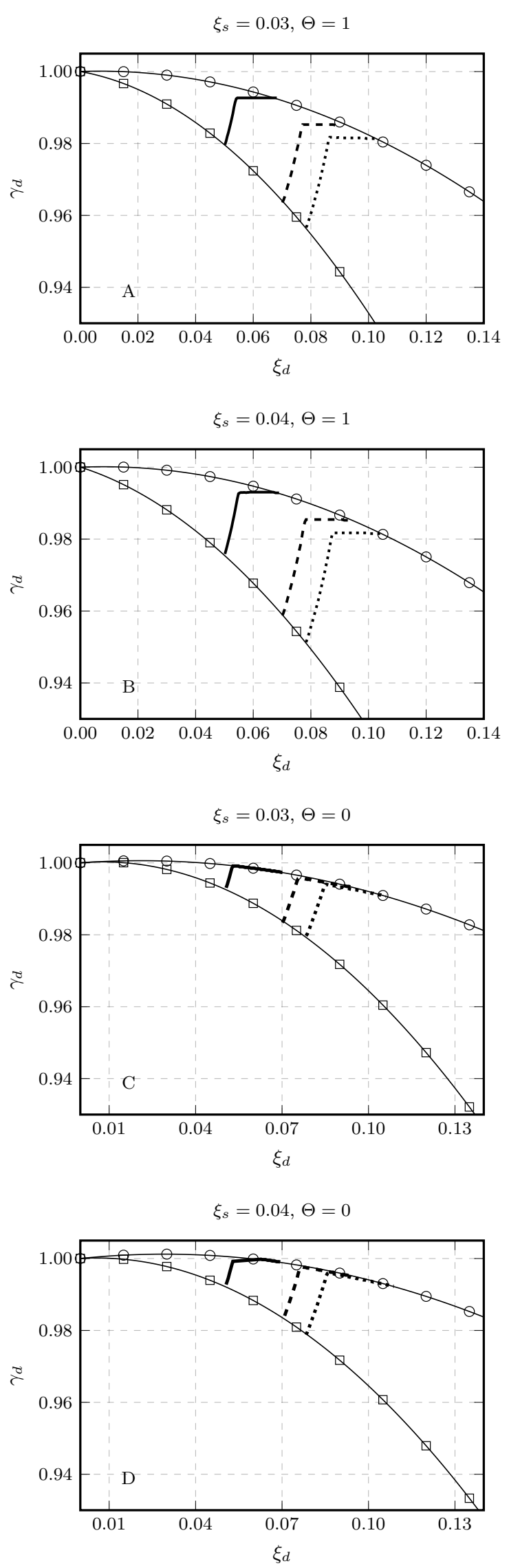

Figure 8. Pareto sets for RMS displacement and peak acceleration reduction for $\xi_{s}=0.03$ and $\xi_{s}=0.04$ under seismic $(\Theta=1)$ and wind induced $(\Theta=0)$ loads. $-\mu_{d}=0.01,--\mu_{d}=0.02, \cdots \mu_{d}=0.025$. $\square$ Optimal design for $f_{2}^{d}$. $\odot$ Optimal design for $f_{\infty}^{a}$. by the optimal design for $f_{\infty}^{d}$ and $f_{\infty}^{a}$, the Pareto set for $F=$ $\left(f_{2}^{d}, f_{\infty}^{a}\right)$ was composed of two segments (Figure 7). In fact, it joined three different points, two of which corresponded to the optimal design for $f_{2}^{d}$ and $f_{\infty}^{a}$. Therefore, in this section, we presented design formulas to find the coordinates of the third point that is the intersection of the segments. Knowing all three points, the Pareto set can be fully characterized.

The values of damping and frequency ratios of the intersection point can be computed by

$$
\begin{aligned}
\xi_{d} & =\sqrt{a_{1} \frac{\mu_{d}\left(1+a_{2} \mu_{d}\right)}{1+\mu_{d}}} \\
\gamma_{d} & =\sqrt{b_{1} \frac{1+b_{2} \mu_{d}}{1+\mu_{d}}}
\end{aligned}
$$

where for seismic excitation

$$
\begin{aligned}
& a_{1}=0.857 \xi_{s}+0.265 \\
& a_{2}=-18.717 \xi_{s}+2.562 \\
& b_{1}=1.021 \xi_{s}^{2}-0.037 \xi_{s}+1.000 \\
& b_{2}=25.217 \xi_{s}^{2}-2.044 \xi_{s}-0.449
\end{aligned}
$$

and under wind induced load

$$
\begin{aligned}
& a_{1}=0.579 \xi_{s}+0.257 \\
& a_{2}=0.339 \xi_{s}+2.897 \\
& b_{1}=2.599 \xi_{s}^{2}+0.097 \xi_{s}+1.000 \\
& b_{2}=4.740 \xi_{s}+0.134
\end{aligned}
$$

In order to derive the design formulas for damping and frequency ratio for the intersection point, we began by approximating $\xi_{d}$ and $\gamma_{d}$ as functions of $\mu_{d}$ for different values of structural damping $\xi_{s}$ under both excitation types using the functions defined in (31) and (32). The considered functoins were found through trial and error. The Least-Squares Fitting was used to find the values of $a_{1}, a_{2}, b_{1}$ and $b_{2}$ for every value of $\xi_{s}$ under seismic and wind induced loads. Afterwards, the values of the control parameters $a_{1}, a_{2}, b_{1}$ and $b_{2}$ as functions of $\xi_{s}$ were approximated by polynomial functions for both excitation types using the same technique.

\section{Discussion and Conclusion}

The multi-objective design of the STMD for the mitigation of structural displacement and acceleration was studied. The main structure was modeled as an SDOF system taking inherent structural damping into account. The main goal of the paper was to find and characterize the set of Pareto optimal damping and frequency ratios of the control device.

This study shows that for the mitigation of structural vibration due to earthquake and induced load considering the $H_{\infty}$ norm, the Pareto set of optimal damping and frequency ratios extends along the frequency ratio, while the damping ratio inside the set is nearly constant (Figure 5). In other words, two Pareto optimal STMD designs can differ greatly in frequency ratio but will have very similar damping ratios. This may be 
interpreted as the fact that the Pareto optimal STMD remains Pareto optimal for some variation in frequency ratio, but not in damping ratio. As such, we can probably conclude that, contrary to the single-objective STMD, the Pareto STMD is sensitive to damping ratio and not to frequency ratio within a certain range.

The single-objective optimization considering the linear combination $f_{\infty}^{d}+f_{\infty}^{a}$ failed (Figure 3 ). This was most likely due to the fact that the Pareto front in this case was linear and the absolute value of the slope was smaller than 1 . In fact, the reduction of peak displacement was higher than that of peak acceleration, which meant that the displacement was more favored during the optimization process. Consequently, the resulting design was optimal for $f_{\infty}^{d}$ only. To get a true optimal design for both objectives, the objective $0.827 f_{\infty}^{d}+f_{\infty}^{a}$ should have been considered. It is therefore not guaranteed for the single-objective optimization of the MOP to always succeed when optimizing for $H_{\infty}$. As a matter of fact, the results will depend on the shape of the Pareto front, and more specifically, its slope.

The outcomes of the present work can provide a convenient method to approximate the Pareto optimal STMD for $F=\left(f_{\infty}^{d}, f_{\infty}^{a}\right)$. As shown through the parametric studies (Figure 5), regardless of the mass ratio, structural damping ratio and excitation type, the Pareto set for the design of the multiobjective STMD was linear and located between the singleobjective optimal designs for displacement and acceleration mitigation. Such designs can be found using existing closed form expressions or single objective optimization. Thus, a linear Pareto set, joining the two designs, might present a good approximation of the actual Pareto set, avoiding the need for combining the design objectives or performing numerical multiobjective optimization.

As for the minimization of RMS displacement $f_{2}^{d}$ and peak acceleration $f_{\infty}^{a}$, the Pareto set was composed of two line segments. The extremities of the set corresponded to the single objective optimal designs for $f_{2}^{d}$ and $f_{\infty}^{a}$. The optimization of the combined objective $f_{2}^{d}+f_{\infty}^{a}$, similar to the previous case, failed. Since the reduction of peak acceleration was higher than the reduction of RMS displacement, $f_{\infty}^{a}$ was favored during the optimization process and the produced design was optimal only for peak acceleration.

For the optimization of $F=\left(f_{2}^{d}, f_{\infty}^{a}\right)$, the Pareto set can be fully described by three points. The two extremities whose coordinates can be computed using existing closed expressions of optimal damping and frequency ratios for $f_{2}^{d}$ and $f_{\infty}^{a}$, or by single objective optimization. Additionally, the coordinates of the third point can be found using the equations presented in the last paragraph of the results.

\section{REFERENCES}

[1] A. Abdelrazaq, Windinduced motion of tall buildings: Designing for occupant comfort, International Journal of High-Rise Buildings, Vol.4, No.1, 2015.

[2] T. E. Saaed, G. Nikolakopoulos, J.E. Jonasson, andH. Hedlund, A state-of-the-art review of structural control systems, Journal of Vibration and Control, Vol.21, No.4, 919-937, 2015.

[3] S. Elias and V. Matsagar, Research developments in vibration control of structures using passive tuned mass dampers, Annual Reviews in Control, Vol.44 No.1, 129$156,2017$.

[4] J.P.D. Hartog, Mechanical Vibrations. Fourth Edition. McGraw Hill, New York, 1956.

[5] G. B. Warburton, Optimum absorber parameters for various combinations of response and excitation parameters, Earthquake Engineering \& Structural Dynamics, Vol.10, No.3, 381-401, 1982.

[6] S. V. Bakre and R. S. Jangid, Optimum parameters of tuned mass damper for damped main system, Structural Control and Health Monitoring, Vol.14, No.3, 448-470, 2007.

[7] J. Salvi and E. Rizzi, Closed-form optimum tuning formulas for passive tuned mass dampers under benchmark excitations, Smart Structures and Systems, Vol.17, No.2, 231-256, 2016.

[8] P. Kamatchi, K. B. Rao, and K. S. Kumar, Closed-form equations for optimum tuning frequency and damping ratio of tuned mass damper and applicability for sitespecific earthquakes, Journal of The Institution of Engineers (India): Series A, Vol.101, No.1, 19-26, 2020.

[9] C. L. Lee, Y. T. Chen, L. L. Chung, and Y. P. Wang, Optimal design theories and applications of tuned mass dampers, Engineering Structures, Vol.28, No.1, 43-53, 2006.

[10] A. Y. Leung and H. Zhang, Particle swarm optimization of tuned mass dampers, Engineering Structures, Vol.31, No.3, 715-728, 2009.

[11] A. Yoyong and H. Muhammad, Optimum placement and properties of tuned mass dampers using hybrid genetic algorithms, International Journal Of Optimization in Civil Engineering, Vol.1, No.1, 167-187, 2011.

[12] M. Ramezani, A. Bathaei, and A. K. Ghorbani-Tanha, Application of artificial neural networks in optimal tuning of tuned mass dampers implemented in high rise buildings subjected to wind load, Earthquake Engineering and Engineering Vibration, Vol.17, No.4, 903-915, 2018.

[13] M. Yucel, G. Bekdas, S. M. Nigdeli, and S. Sevgen, Estimation of optimum tuned mass damper parameters via machine learning, Journal of Building Engineering, Vol.26, 100847, 2019.

[14] K. Xu and T. Igusa, Dynamic characteristics of multiple substructures with closely spaced frequencies, Earthquake Engineering \& Structural Dynamics, Vol.21, No.12, 1059-1070, 1992. 
[15] O. Lavan, Multiobjective optimal design of tuned mass dampers, Structural Control and Health Monitoring, Vol.24, No.11, e2008, 2017.

[16] S. Etedali and H. Rakhshani, Optimum design of tuned mass dampers using multiobjective cuckoo-search for buildings under seismic excitations, Alexandria Engineering Journal, Vol.57, No.4, 3205-3218, 2018.

[17] M. Dellnitz, O. Schutze, and T. Hestermeyer, Covering Pareto sets by multilevel subdivision techniques, Journal of Optimization Theory and Applications, Vol.124, No.1, 113-136, 2005.
[18] Mogensen, Patrick Kofod and Riseth, Asbjørn Nilsen, Optim: A mathematical optimization package for Julia, Journal of Open Source Software, Vol.3, No.24, 615, 2018.

[19] Steven G. Johnson, The NLopt nonlinear-optimization package, http://github.com/stevengj/nlopt.

[20] N. Jorge and W. Stephen, Numerical Optimization, Second Edition, Springer, New York, 2006. 